\title{
An augmented level set model for the propagation of bushfire fronts
}

\author{
$\underline{\text { S. Berres }}^{\mathrm{a}}$ and N. Cárcamo ${ }^{\mathrm{a}}$ \\ a Departamento de Ciencias Matemáticas y Físicas, Universidad Católica de Temuco, Temuco, Chile \\ Email: sberres@uct.cl
}

\begin{abstract}
Based on the model of Rothermel (1972), during several decades, the spread of bushfires has been studied by empirical, quasi-empirical and physical models, in order to increase the knowledge on this phenomenon and its relevant characteristics, and to deliver timely information for fire extinction. In Chile, since 1976, the UNESCO has proclaimed in total 9 Biosphere Reserves within its 'Man and the Biosphere' program (Gedda, 2014). This program emphasizes the importance of protection measures and the relevance of scientific research in the area.

Recently, in March 2015, a large forest fire in the 'China Muerta' National Park in mid-southern Chile devastated some 8,000 hectares of native Araucaria araucana-Nothofagus forest, as firefighters had a hard job to extinguish the fires. This study is motivated by the need of a better understanding of forest fire dynamics in order to allow for improved prediction and prevention measures.

Propagating fronts or interfaces in general can be described by the Hamilton-Jacobi equation

$$
\frac{\partial \phi}{\partial t}+v \frac{\partial \phi}{\partial x}+w \frac{\partial \phi}{\partial y}=0
$$

where $\phi=\phi(t, x, y)$ represents the front implicitly as level-set where $\phi=0$. This implicitly defined front is propagated by the convection velocities $v$ and $w$ in $x$ and $y$ direction, respectively. The propagation direction is normal to the front, pointing to the side where $\phi>0$. Propagating fronts can be observed in different situations such as the interaction of gas and liquid in improved oil recovery, medical image segmentation and object tracking.

In the application of the Hamilton-Jacobi equation to bushfire propagation, the sign of $\phi$ distinguishes whether the corresponding area is burned $(\phi<0)$ or unburnt $(\phi>0)$, thus implicitly characterising the location of the fire front as $\phi=0$.

In this contribution, an augmented propagation model based on the Hamilton-Jacobi equation is developed by introducing additional variables such as combustion and heat. The evolution of these variables is described by differential equations. The goal is to couple the equation for front evolution with a heat equation and an equation that corresponds to the local combustion process. The model dynamics is demonstrated by numerical simulations.
\end{abstract}

Keywords: Hamilton-Jacobi equation, level set method, bushfire front propagation 
S. Berres and N. Cárcamo, An augmented level set model for the propagation of bushfire fronts

\section{INTRODUCTION}

Bushfire propagation models are formulated to be able to gather and consider prior information, which can be useful in an emerging fire attack. Studies focussing on specific fire events have yielded valuable insights that certain parameters like soil properties, combustion material, moisture or slope characterize the physical phenomenon, see e.g. Pedernera et al. (2010), Cassagne et al. (2011), Dumond (2009), Alexander and Cruz (2013), Hilton et al. (2015). Complementarily, models originated as a rather academic exercise, even though they do not fully describe the whole phenomenon, have shown to find their way to operational or experimental use, see Sullivan (2007).

Since the 1920s, various empirical, quasi-empirical and physical propagation models have been proposed. Several authors argue that for fire spread there are three factors that have a significant influence, namely topography, climate and fuel, see Rothermel (1972), Sethian (1996), Finney (2004), Sullivan (2007). A general consensus is also the ellipsoidal form for the spread of fire, although other forms as ovals or drops may be more accurate according to the scenario or fuel type being studied (Dumond (2009); Finney (2004); Hilton et al. (2015), Mallet et al. (2009)). We also refer to coupled fire-atmosphere models, where the fire spread model includes fuel decay and heat release, see Mandel et al. (2011).

The forest fuel consists of living and dead plant material as shrubs, wood or grass, i.e. biomass consisting of cellulose as main chemical component (Sullivan, 2007). The principal physical process during burning is heat transfer, which can be realized through conduction, convection or radiation.

This contribution adapts the setup of using a Hamilton-Jacobi equation (1), and in particular the Level Set Method proposed by Osher and Fedkiw (2006), as modeling framework for the numeric calculation of fire front propagation. Thereby, the movement of the interface is described by an Eulerian formulation, where the function $\phi$ represents the front implicitly as the set satisfying $\phi=0$.

(a)

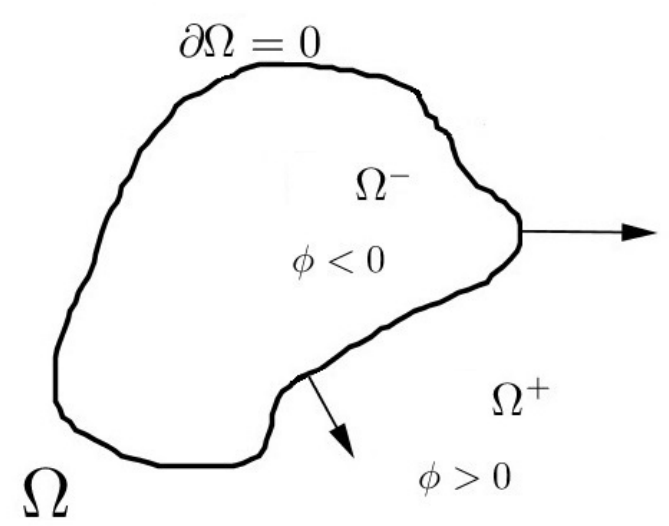

(b)

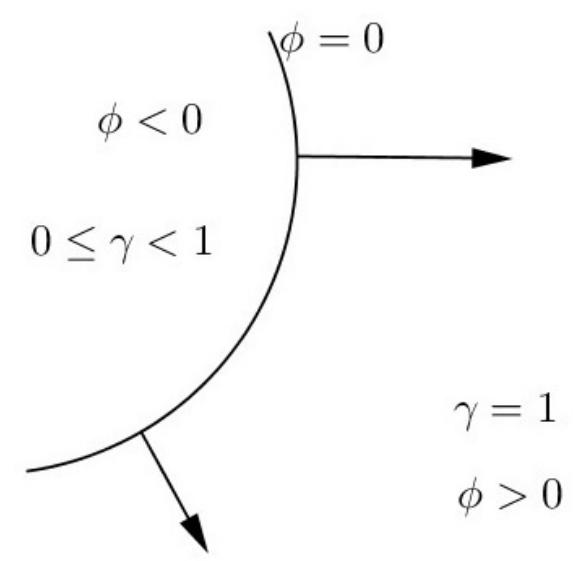

Figure 1. (a) Partition of $\Omega=\Omega^{-} \cup \partial \Omega \cup \Omega^{+}$corresponding to the subdomains where $\phi<0, \phi=0$ and $\phi>0$, respectively. (b) Values of combustible $\gamma$ : full load $\gamma=1$ in unburnt area $(\phi>0)$, reduced combustible $\gamma \in(0,1)$ in burning area $(\phi<0)$.

Figure 1 (a) explains the partition of the two-dimensional spatial domain $\Omega$ for distinct values of $\phi$, namely $\partial \Omega=\{(x, y) \mid \phi=0\}$ for the fire front itself, $\Omega^{+}=\{(x, y) \mid \phi>0\}$ for the unburnt surface, and $\Omega^{-}=$ $\{(x, y) \mid \phi<0\}$ for the burning or burnt surface.

Combustion is a chemical process with fuel and oxygen as main ingredients. Given that these ingredients are exhaustively available in forests or bushland, fires occur naturally as soon as the ignition temperature of the respective fuel is reached. In order to track the fuel change in a fire, the variable $\gamma$ is introduced to account for the fuel amount, which evolves according to the combustion rate. The fuel amount $\gamma$ depends on the fire status $\phi$ as

$$
\gamma\left\{\begin{array}{llll}
=1, & \text { for } \phi>0, & \text { (unburnt surface) } \\
\in & {[0,1)} & \text { for } \quad \phi<0, & \text { (burning surface) }
\end{array}\right.
$$


S. Berres and N. Cárcamo, An augmented level set model for the propagation of bushfire fronts

Here, $\gamma=1$ corresponds to full fuel load, which applies for the unburnt area (where $\phi>0$ ), and $\gamma \in$ $[0,1$ ) indicates reduced combustible, which applies for the burning area (where $\phi<0$ ), see Figure 1 (b) for illustration. In the augmented model, the fuel $\gamma$ and the resulting heat are incorporated as additional variables, which are described by corresponding equations, that eventually take account of environmental conditions.

\section{MODELLING}

In the modelling of bushfire spread, the most relevant physical properties are fuel moisture, topographic characteristics or weather conditions. The conceptual modelling indicates how these properties can be included in a mathematical modeling framework set up by the Hamilton-Jacobi equation that describes the fire front propagation.

\subsection{Conceptual modelling}

In the spread of bushfires the propagation speed depends on how the heat received by the fuel is related to the heat required to bring the fuel to the ignition temperature which activates the combustion. The equation of Rothermel (1972)

$$
R=\frac{I_{R} \xi\left(1+\phi_{w}+\phi_{s}\right)}{\rho_{b} \zeta Q_{\mathrm{ig}}}
$$

predicts the propagation speed $R$ in a bed of homogeneous fuel by experimental data, using statistical correlation techniques for adjustments.

The topography is expressed in terms of the slope of the surface, which is flat under ideal conditions. Rothermel (1972) defined a dimensionless coefficient $\phi_{s}=5.275 \beta^{-0.3}(\tan \phi)^{2}$ with packing ratio of the fuel bed $\beta$, assuming that there is no relationship between slope $\tan \phi$ and wind direction. In the work of Finney (2004), the effects of varying slope and wind combinations establishing a so-called 'wind-slope vector' are simulated. In their documented simulation, some interesting effects can be observed: The length of the spread increases disproportionately with the wind speed. For example, the spread of the fire at wind speed of $20 \mathrm{~m} / \mathrm{s}$ is more than the double than the spread at wind speed $10 \mathrm{~m} / \mathrm{s}$.

The topography directs how energy is transferred to the fuel and the atmosphere. The upslope area is burned faster than the downward slope area, see Sullivan (2007).

For the wind being a relevant factor, Rothermel (1972) defined a dimensionless coefficient given by $\phi_{w}=$ $C U^{B}\left(\frac{\beta}{\beta_{o p}}\right)^{-E}$, where $\beta_{o p}$ is the fuel packing for optimal combustion, $U$ is the mean wind velocity at midflame height, and $C, B$ and $E$ are parameters depending on the fuel bed.

In the conceptual modeling of the physical phenomenon we consider the interaction of various variables such as heat $\eta$, fuel $\gamma$ and the fire state $\phi$, in addition to parametric functions as environmental conditions $a$ and combustion rate $b$.

\subsection{Mathematical modelling}

Essentially, there are three modelling variants to describe the movement of a front positioned at points $(x, y)$ propagating at velocities $(v, w)$, namely a Lagrangian, an Eulerian, and a hybrid modelling approach. These modeling approaches correspond, respectively, to the situations where the positions $(x, y)$ are dependent or independent variables, or are split, one dependent and the other independent.

The Lagrangian formulation

$$
\frac{d}{d t}\left(\begin{array}{l}
x \\
y
\end{array}\right)=\left(\begin{array}{l}
v \\
w
\end{array}\right)
$$

describes the movement of each point $(x, y)$, being dependent variables, on the front to have the velocities $(v, w)$, where the set of points on the front can be parametrized by arc length.

In the hybrid formulation in terms of the convection-reaction equation

$$
\frac{\partial y}{\partial t}+v \frac{\partial y}{\partial x}=w
$$


S. Berres and N. Cárcamo, An augmented level set model for the propagation of bushfire fronts

the position $y=y(t, x)$ of the front is a dependent variable, expressed as a function of the independent variable $x$. In the equation, the propagation speed $v$ in $x$ direction appears as the convection velocity of the $y$-profile, whereas the speed $w$ in $y$ direction turns out to be the reaction term of the equation. Such a hybrid modelling approach has been adopted e.g. by Sharples et al. (2013) to the modeling of fire front propagation.

In Eulerian coordinates, front propagation can be expressed in terms of the Hamilton-Jacobi equation (1). In this modeling approach the positions $(x, y)$ are independent variables. The front position is described implicitly by $\phi=0$. Convection velocities $(v, w)$ in $(x, y)$ direction are defined for any location, and in particular for the points on the front. In the modelling of fire front propagation the variable $\phi$ describes the fire status, positive for burning area, negative for unburnt area, such that $\phi=0$ describes the fire front.

The fire front modelling can be adapted for each of these three approaches. The focus of this contribution is on the Eulerian approach. Within the Eulerian modelling framework the convection velocities are incorporated as follows. The normal vector for points on the front is defined as

$$
\mathbf{n}=\frac{\nabla \phi}{\|\nabla \phi\|_{2}}, \quad \nabla \phi=\left(\phi_{x}, \phi_{y}\right)^{\mathrm{T}} .
$$

If the normal vector (5) indicates the direction of the front propagation and some parameter $a$ the speed, then the velocity vector $U=(v, w)^{\mathrm{T}}$ becomes

$$
U=\left(\begin{array}{c}
v \\
w
\end{array}\right)=a \mathbf{n}, \quad v=\frac{a}{\|\nabla \phi\|_{2}} \phi_{x}, \quad w=\frac{a}{\|\nabla \phi\|_{2}} \phi_{y} .
$$

Here, the speed $a=a(x, y, t)$ is not necessarily a constant but might depend the context, reflecting spatial and temporal influences. Substituting (6) in the convective terms of the Eulerian model (1), by the definition of the Euclidean norm one gets

$$
v \phi_{x}+w \phi_{y}=\frac{a}{\|\nabla \phi\|_{2}}\left(\phi_{x}^{2}+\phi_{y}^{2}\right)=\frac{a}{\|\nabla \phi\|_{2}}\left(\|\nabla \phi\|_{2}\right)^{2}=a\|\nabla \phi\|_{2},
$$

which in turn gives the governing equation

$$
\phi_{t}+a\|\nabla \phi\|_{2}=0
$$

This is an equation that describes the evolution of implicitly defined curves. It can be resolved numerically with methods proposed by Osher and Sethian (1988).

(a)

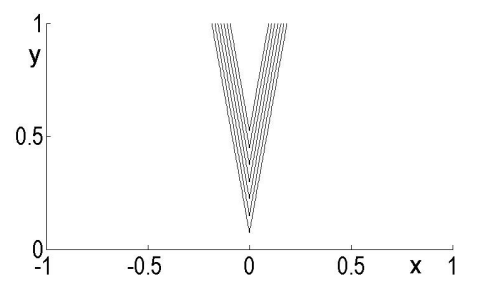

(b)

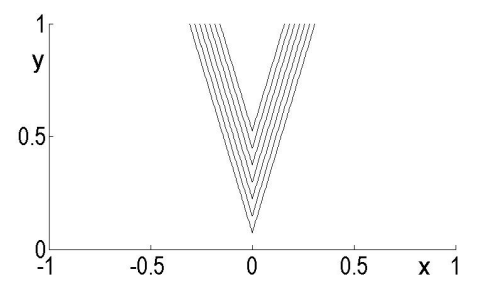

(c)

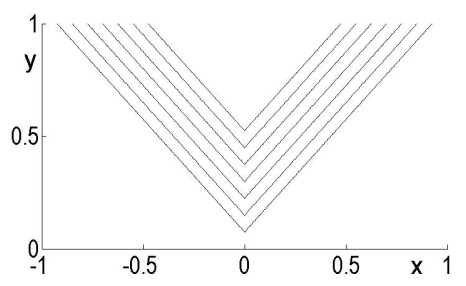

Figure 2. Propagation of level set zero of $\phi$ with initial data $\phi=y-r|x|$, (a) $r=5$, (b) $r=3$, (c) $r=1$.

We consider as sample situation the interaction of two fire fronts at a given angle, see Sharples et al. (2013). In terms of $\phi$, the initial condition is expressed as

$$
\phi=y-r|x| \text {. }
$$

Figure 2 shows the front evolution according to equation (8) with $a=1$. In the initial condition (9) the parameter values $r \in\{1,3,5\}$ correspond to different front inclinations. The propagation occurs without considering curvature effects as mentioned in Sharples et al. (2013). 


\subsection{Augmented model}

The augmented model is obtained by incorporating some relevant aspects of the physical phenomenon, which are identified by the conceptual model, into the chosen mathematical modelling framework. In the present application context we have identified the available fuel and the produced heat to be elegible concepts that are suited to be formalized by choosing them as variables of an augmented model. Thus, the governing equation (8) is coupled with equations for the fuel $\gamma$ and the heat $\eta$.

The combustion process is described by the differential equation

$$
\frac{d \gamma}{d t}=-b \gamma, \quad \gamma(t=0, x, y)=1, \quad \text { for } \quad(x, y) \in \Omega,
$$

which is defined for all locations in the two-dimensional spatial domain $\Omega$. A similar approach of ignition modeling, where the fuel is a variable of a coupled modelling approach, is done in Mandel et al. (2011). However, there, the fuel evolution is not explicitly described by a differential equation; rather, the fuel amount is given by a formula that looks like a solution of a linear differential equation.

The initial condition $\gamma(0, x, y)=1$ corresponds to a full fuel load. The fuel load is largest up to ignition, and it decreases as the fuel is burning. According to the model formulated in equation (10), the right-hand side is proportional to the remaining fuel. The combustion rate $b=b(x, y, t)$ might depend on external effects such as rain, moisture or fuel type. In this contribution, it is assumed that these external effects are homogenous having a constant combustion rate $b_{0}>0$ at all locations with ongoing burning,

$$
b(x, y)=\left\{\begin{array}{cc}
b_{0} & \text { if } \quad \phi(x, y)<0, \\
0 & \text { if } \quad \phi(x, y)>0,
\end{array} \quad \text { for } \quad(x, y) \in \Omega\right.
$$

During the combustion processes, energy is released as heat or light. The heat evolution is modeled as

$$
\frac{\partial \eta}{\partial t}=k\left(\frac{\partial^{2} \eta}{\partial x^{2}}+\frac{\partial^{2} \eta}{\partial y^{2}}\right)+b c \gamma-d\left(\eta-\eta^{0}\right) .
$$

This equations counts with a term for heat production by combustion, which is proportional to the burning rate according to equation (10). If the fuel amount $\gamma$ is closer to 1 , then there is larger fuel diminution by combustion (10), and at the same time there is a larger heat production in (12) through the term $b c \gamma$. A maximal temperature is given implicitly by the limited heat potential of the fuel, $\int_{0}^{\infty} \gamma(t) d t<\infty$. It is obtained when heat production and heat diffusion are balanced.

\section{COMPUTATIONAL SIMULATION}

For the numerical solution of the Hamilton-Jacobi equation (1), as numerical flux, the local Lax-Friedrichs flux was used together with an ENO reconstruction of order 3. The time discretization was done with an explicit Euler method. To ensure stability of the explicit scheme the so-called CFL condition is satisfied. During the simulation with the level set method, a usual technique to maintain the accuracy of the approximate solution is to perform regularly a re-initialziation step. However, in the simulations of this contribution, this turned out to be not necessary. For the discretization of the diffusion, the use of the Crank-Nicolson method is a standard approach. However, the test cases considered in this contribution do not involve the diffusion term. A detailed study of the effect of the modelled heat diffusion is going to be provided in an extended version.

In the augmented model, the equation (8) is coupled with the fuel equation (10) and the heat evolution according to equation (12). For the simulations of the augmented model, the parameters are set to hypothetical values, which give qualitatively and quantitatively reasonable simulation results. The front propagation speed in (8) is set to $a=1$. For the initial condition (9) we set $r=1$. In order to focus on the reaction dynamics we assume the situation without heat conduction, i.e. $k=0$. The temperature of the environment is set to $\eta^{0}=20$, which also is used for the initial condition, $\eta(t=0, x, y)=\eta^{0}$ for the non-burning area, where $\phi(t=0, x, y)>0$. For the burning area we set $\eta(t=0, x, y)=\eta^{\text {ign }}$ if $\phi(t, x, y)<0$, where $\eta^{\text {ign }}$ is the ignition temperature of the fuel, here we take $\eta^{\text {ign }}=500$.

The heat absorption by the atmosphere is scaled by $d=50$. From the conceptual model it is deferred that the temperature increase depends on the heat radiated by combustion. Therefore, we set the corresponding heat release factor as $c=4500$. The fuel decay rate due to combustion is set to $b_{0}=10$. 
S. Berres and N. Cárcamo, An augmented level set model for the propagation of bushfire fronts

In Figure 3, the fuel and heat distribution at intermediate time $t=0.5$ are shown for varying $x$ and $y$. The fuel load $\gamma$ appears to obey a Laplace-type distribution, which comes from the analytical solution of equation (10). The fact that the distribution is expressed in terms of $x$ instead of $t$ can be explained by the constant convection speed in equation (8), which impacts the combustion via (11).

(a)

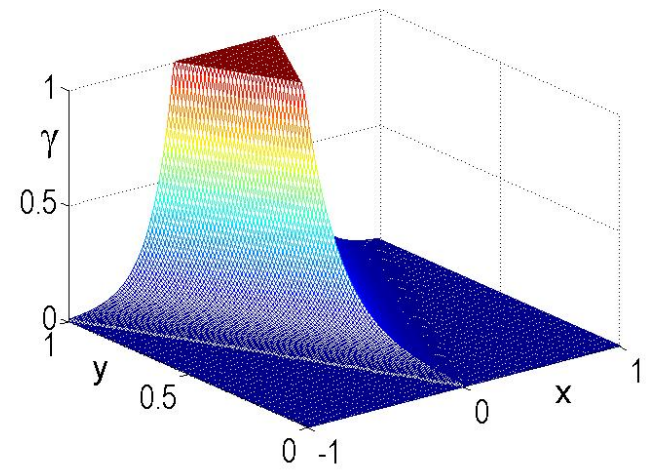

(b)

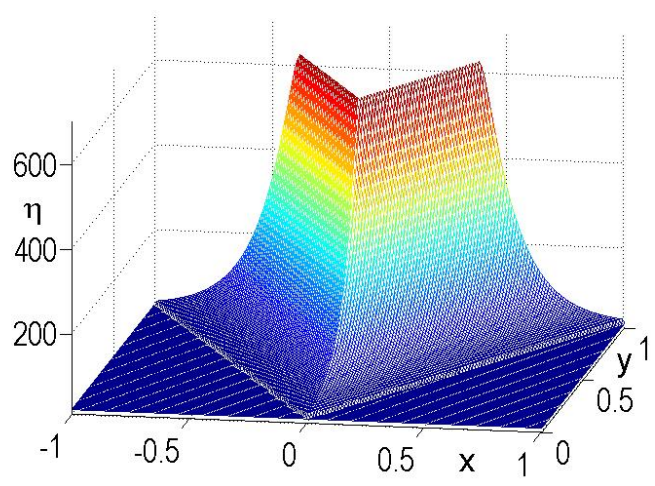

Figure 3. Magnitude of variables in domain $\Omega$ at intermediate time $t=0.5$, (a) fuel load $\gamma$, (b) heat $\eta$.

Figure 4 shows selected one-dimensional profiles of the simulation data. The fuel load $\gamma$ and heat $\eta$ at various intermediate times are shown for fixed $x=0.25$ and varying $y \in[0,1]$. It can be observed that the heat maximum is centered near the fire front.

(a)

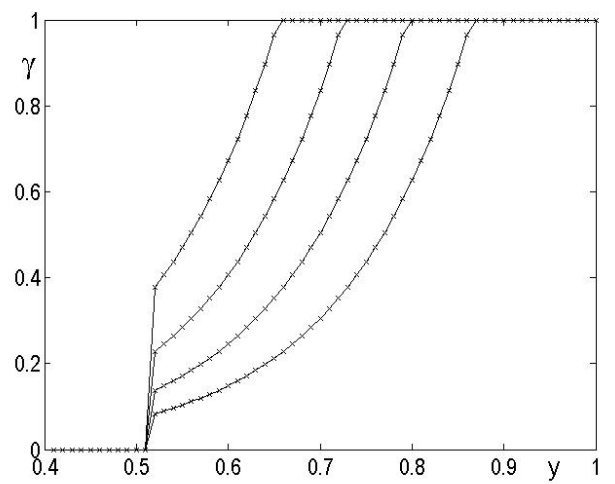

(b)

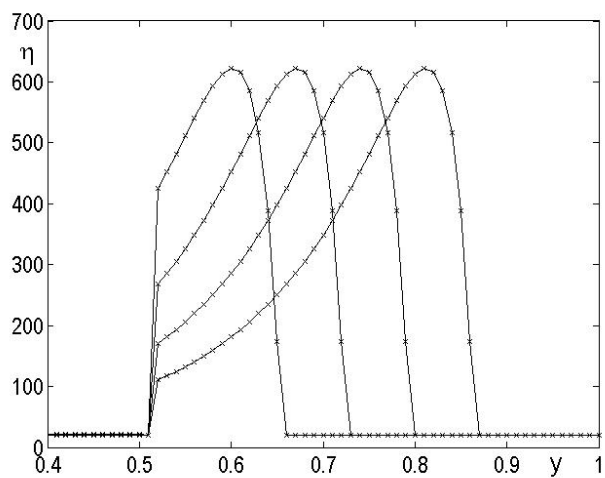

Figure 4. Solution profiles for $x=0.25$ at times $t \in\{0.1,0.15,0.2,0.25\}$, (a) fuel load $\gamma$, (b) heat $\eta$.

\section{DISCUSSION}

In this contribution, the propagation of bushfire is modeled by a Hamilton-Jacobi equation coupled with two additional equations that describe the fuel and heat evolution, where parametric functions describing environmental conditions and the combustion rate. The model dynamics is illustrated by numerical simulations. A pending task is, besides running further test cases and parameter studies, the comparison with experimental data and the outcomes of other simulations tools.

The coupling of equations (8), (10) and (12), as stated here, is one-way: the front evolution and thus the fire location affects the fuel change and thus the heat release, but the heat release does not affect reversely the front evolution. However, in real-world scenarios it is expected that the heat also should affect the spread reate. In a further model development, one desired feature is that the propagation velocity $a$ depends on $\eta$, in the sense that a higher temperature leads to a faster propagation. This can be achieved by starting with a linear approach, setting $a=a(\eta)=a_{0}+a_{1} \eta$ with $a_{0}, a_{1}>0$.

The heat is evolved according to a diffusion term with diffusivity $k$. Besides a temperature evolution in the $(x, y)$ directions of the landscape, a model that considers heat evolution is expected to account for a 
S. Berres and N. Cárcamo, An augmented level set model for the propagation of bushfire fronts

temperature radiation into the atmosphere in $z$-direction, eventually counting with a three-dimensional heat diffusion operator $\Delta \eta=\eta_{x x}+\eta_{y y}+\eta_{z z}$. However, since a coupling with an atmosphere model is not intended within this contribution, the heat diffusion in $z$ direction is modelled by a heat absorption term. For extensive bushfire-atmosphere coupling see Mandel et al. (2011).

A boundary condition corresponding to the $z$-direction has to be set anyway, independently whether it consists of one single or several layers. We opt to describe the atmosphere as one single layer, such that the atmospheric cooling is modelled by the $d\left(\eta-\eta^{0}\right)$ lumped term on the right hand side of equation (12). This corresponds to a temperature disminiuition by absorption through the environment, where the reference temperature of the environment is $\eta^{0}$. A compromise for the inclusion of effects in $z$ direction would be to model the atmosphere by a limited amount (e.g. around 3) layers.

\section{ACKNOWLEDGEMENT}

This article is a product of the 'Observatorio Regional UC Temuco' in the context of the 'Convenio de Desempeño para la Educacin Superior Regional UCT1302’.

\section{REFERENCES}

Alexander, M. E. and M. G. Cruz (2013). Are the applications of wildland fire behaviour models getting ahead of their evaluation again? Environmental Modelling \& Software 41, 65-71.

Cassagne, N., F. Pimont, J.-L. Dupuy, R. R. Linn, A. Mårell, C. Oliveri, and E. Rigolot (2011). Using a fire propagation model to assess the efficiency of prescribed burning in reducing the fire hazard. Ecological Modelling 222, 1502-1514.

Dumond, Y. (2009). An applied step in forest fire spread modelling. In ECMS, pp. 32-38.

Finney, M. A. (2004). Farsite: Fire area simulator: model development and evaluation.

Hilton, J., C. Miller, A. Sullivan, and C. Rucinski (2015). Effects of spatial and temporal variation in environmental conditions on simulation of wildfire spread. Environmental Modelling \& Software 67, $118-127$.

Mallet, V., D. Keyes, and F. Fendell (2009). Modeling wildland fire propagation with level set methods. Computers \& Mathematics with Applications 57, 1089-1101.

Mandel, J., J. Beezley, and A. Kochanski (2011). Coupled atmosphere-wildland fire modeling with WRF 3.3 and SFIRE 2011. Geosci. Model Dev. 4, 591-610.

Osher, S. and R. Fedkiw (2006). Level set methods and dynamic implicit surfaces, Volume 153. Springer Science \& Business Media.

Osher, S. and J. A. Sethian (1988). Fronts propagating with curvature-dependent speed: Algorithms based on Hamilton-Jacobi formulations. Journal of Computational Physics 79, 12-49.

Pedernera, P., M. Castillo, and G. Julio (2010). Diseño y validacion de un simulador de incendios forestales en Chile.

Rothermel, R. C. (1972). A mathematical model for predicting fire spread in wildland fuels.

Sethian, J. A. (1996). A fast marching level set method for monotonically advancing fronts. Proceedings of the National Academy of Sciences 93, 1591-1595.

Sharples, J., I. Towers, G. Wheeler, V.-M. Wheeler, and J. McCoy (2013). Modelling fire line merging using plane curvature flow. In J. Piantadosi, R. Anderssen, and J. Boland (Eds.), MODSIM2013, 20th International Congress on Modelling and Simulation. Modelling and Simulation Society of Australia and New Zealand, pp. 256-262.

Sullivan, A. (2007). A review of wildland fire spread modelling, 1990-present, 1: Physical and quasiphysical models. arXiv preprint arXiv:0706.3074. 